\title{
Structural modifications during conducting polymer formation — an ellipsometric study
}

\author{
L.M. Abrantes ${ }^{\mathrm{a}, \mathrm{b}, *}$, J.P. Correia ${ }^{\mathrm{b}, \mathrm{c}}$, M. Savic ${ }^{\mathrm{b}}$, G. Jin ${ }^{\mathrm{d}}$

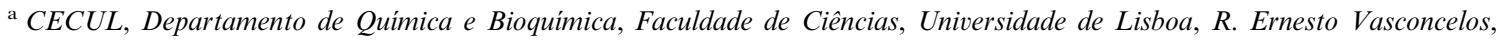 \\ 1749-016 Lisbon, Portugal \\ ${ }^{\mathrm{b}}$ ICAT, Laboratório de Processos Electroquímicos, Campo Grande, 1749-016 Lisbon, Portugal \\ ${ }^{\mathrm{c}}$ INETI, Departamento de Energias Renováveis, 1649-038 Lisbon, Portugal \\ d Institute of Mechanics, Chinese Academy of Sciences, 15, Zhong-Guan-Cun Road, Beijing 100080, China
}

Received 20 November 2000; received in revised form 28 February 2001

\begin{abstract}
Polyaniline films potentiodynamically synthesised on gold substrates are analysed ex situ by conventional and imaging ellipsometry (at constant wavelength). Polymers grown up to 50 cycles appear to be homogeneous, whereas different structures are displayed by thicker layers. The memory effect, induced by the polymer electrochemical characterisation, in subsequent polymer growth, is demonstrated. In the case of thin films, a reliable methodology has been successfully employed to obtain, from the ellipsometric parameters $\Delta$ and $\psi$, the refractive index, the extinction coefficient and the thickness of polymer layers. This approach also allowed us to quantitatively evaluate the magnitude of polymer swelling upon oxidation (11-22\%). Furthermore, the surface morphology is well evidenced by the results obtained by imaging ellipsometry. (C) 2001 Elsevier Science Ltd. All rights reserved.
\end{abstract}

Keywords: Polyaniline; Ellipsometry; Polymer modified electrodes; Optical properties; Polymer structure

\section{Introduction}

Electrode surfaces modified by layers of conducting polymers have attracted a great deal of attention in the last decade [1-3]. Due to fundamental scientific interest and the possible technological applications of polyaniline (PANI) it has been one of the most studied materials, and polymer film preparation $[4,5]$ as well as other properties [6,7] have been well reported.

Electrochemical polymerisation, usually performed on inert metallic electrodes (e.g. Pt or Au) is a widely used method for conducting polymer film synthesis.

\footnotetext{
* Corresponding author. Tel.: + 351-21-7500-016; fax: + 351-21-7500-115.

E-mail address: luisa.abrantes@cd.fc.ul.pt (L.M. Abrantes).
}

This route presents several advantages such as one step polymerisation, highly conducting film deposition and doping and thickness control via electrode potential. However, results gathered from electropolymerisation carried out under a variety of experimental conditions clearly show the influence of the growth parameters on the properties of the obtained polymer films [8-10].

In the case of PANI, it is generally accepted that multi-sweep cyclic voltammetry is the most adequate approach to prepare homogeneous conducting films [11,12]; Cruz and Ticianelli [13] have recently published a systematic electrochemical and ellipsometric study of PANI deposition under potentiodynamic conditions which reveals polymer structural transitions in several stages of growth. The authors have considered that after a critical thickness there is a significant change in the PANI growth mechanism. 
A gradual transformation from ordered crystalline to amorphous nodular material was earlier reported by Yang et al. [14] for polypyrrole and polythiophene electrodeposition on graphite electrodes, based on scanning tunnelling microscopy data. Tian et al. [15] have also used ex situ ellipsometry to determine the poly(3methylthiophene) structure at several stages of deposition, and a thin dense fibrous layer followed by a thick granular more open structure have been described. Therefore, it appears that a non-uniform growth process shall be considered a common feature in the electropolymerisation route.

Differences in the polymer structure upon thickening shall influence the swelling and deflation of fibres upon doping/undoping process. Although a thorough understanding of the phenomena is central for the control and performance optimisation of the physical properties of PANI, the available explanation is not yet satisfactory.

The purpose of the present work is to study the structural properties of PANI films of different thickness potentiodynamically prepared on gold, under the same conditions. Polymer growth experiments were designed to avoid perturbations from the amplitude of the applied potential: the anodic limit has been progressively decreased during polymerisation to account for the monomer oxidation potential shift in the negative direction as the chain increases, and thus to maintain the progress of the reaction.

Since conventional ellipsometry has proven to be a useful approach for the characterisation of conducting polymer films electrochemically grown on metals, the technique was employed, in constant wavelength mode, for the determination of film refractive index $(\tilde{n}=n-$ $\mathrm{i} k$ ) in the oxidised and reduced forms.

Based on the ellipsometric principle a simple optical imaging system for visualisation of surfaces with layer thickness patterns has been recently reported [16,17]. It permits to carry out an ellipsometric analysis point by point in a large area of illumination.

The imaging method has been used in this work to obtain the thickness distribution (with $0.3 \mathrm{~nm}$ resolution) of PANI layers. The optical properties of the deposits point to a homogeneous PANI growth up to $50 \mathrm{~nm}$, displaying thereafter different structures.

The ellipsometric measurements were performed ex situ in order to obtain the optical data, by both the ellipsometric techniques, from the films in the same conditions (since the imaging ellipsometry set-up does not allow in situ measurements).

The valuable complementary information provided by ellipsometry is also revealed by a successfully employed methodology to obtain the thickness of thin PANI films from the experimental parameters phase, $\Delta$, and azimuth, $\psi$.

\section{Experimental}

From a solution containing $0.1 \mathrm{M}$ aniline in $0.5 \mathrm{M}$ $\mathrm{H}_{2} \mathrm{SO}_{4}$, the PANI films were potentiodynamically grown by cycling the potential between -0.2 and $+0.8 \mathrm{~V}$ (vs. saturated calomel electrode) at a sweeping rate of $50 \mathrm{mV} \mathrm{s}^{-1}$; after five cycles the anodic limit was reduced to 0.75 and to $0.7 \mathrm{~V}$ after eight, to lessen the possibility of producing undesirable by-products.

The electrolyte solutions were prepared from aniline distilled under reduced pressure prior to use and 'Aristar' grade sulphuric acid in Millipore water. They were thoroughly deoxygenated directly in the cell with argon (purity > 99.9997\%) and an inert atmosphere was kept over the solution surface during the measurements. All experiments were performed at room temperature (23$\left.24{ }^{\circ} \mathrm{C}\right)$.

A three-electrode cell was employed to perform the electrochemical experiments being the working electrode potential controlled with respect to SCE. The working electrodes consist of gold evaporated chromium coated silicon slides $\left(\mathrm{Si}, \mathrm{SiO}_{2}(2 \mathrm{~nm}), \mathrm{Cr}\right.$ (2 $\mathrm{nm}), \mathrm{Au}(100 \mathrm{~nm}))$ and a large area platinum foil was used as counter electrode.

PANI films with 20, 50 and 100 growth cycles were synthesised. A 'stair' sample was also prepared consisting of a film with 20 cycles and another 30 polymerisation cycles in half of the surface of the first layer, after its electrochemical characterisation.

The films redox behaviour were studied by cyclic voltammetry in a monomer free solution in the potential range between -0.2 and $+0.5 \mathrm{~V}$ at a scan rate of $50 \mathrm{mV} \mathrm{s}^{-1}$. After the electrochemical characterisation, the electrodes were thoroughly rinsed with Millipore water and dried under a flux of argon. Due to the potential shift that occurs when the polarisation of the electrode is interrupted, the so-called oxidised and reduced state, in this work, does not correspond to a fully doped or undoped PANI film but instead to the polymers at open circuit potentials after being subjected to potentials of 0.5 and $-0.2 \mathrm{~V}$, respectively, for $5 \mathrm{~min}$.

Ex situ conventional ellipsometric characterisation of the PANI films was carried out in a rotating analyser type Sentech ellipsometer equipped with a $\mathrm{He}-\mathrm{Ne}$ laser $(\lambda=632.8 \mathrm{~nm})$ working with an angle of incidence of $70^{\circ}$.

The imaging ellipsometry experiments were performed using an expanded light beam instead of the conventional narrow one. The source is a Xenon arc lamp with a $633 \mathrm{~nm}$ interference filter (bandwidth of 10 $\mathrm{nm}$ ) and a collimating system to ensure a uniform intensity distribution over the beam area. The photodetector consists of a CCD video camera $(752 \times 582$ pixels) allowing more than $4 \times 10^{5}$ points in an area of $15 \times 25 \mathrm{~mm}^{2}$ to be simultaneously analysed. The measurements were performed at an incident angle close to 


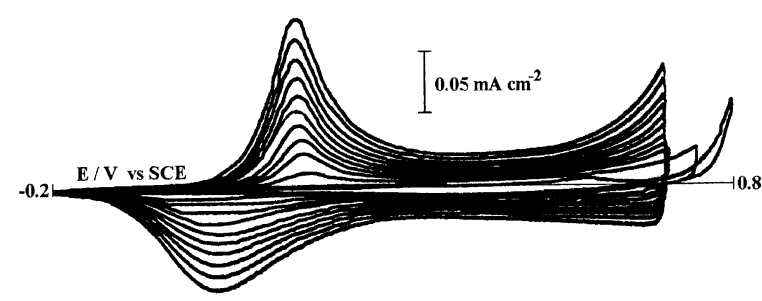

Fig. 1. Electrochemical growth of PANI film on a gold coated silicon electrode. $0.1 \mathrm{M}$ aniline in $0.5 \mathrm{M} \mathrm{H}_{2} \mathrm{SO}_{4} ; v=50$ $\mathrm{mV} \mathrm{s}^{-1}$.

the pseudo-Brewster angle of the substrate. Fulfilling the null reading conditions for the bare gold surface, the thickness of the different regions of the film is proportional to the square root of the reflected light intensity [17].

\section{Results and discussion}

Fig. 1 shows the cyclic voltammograms recorded during the aniline polymerisation. The thickening of the film is evidenced by the continuous increase of the height of the polymer redox peaks. The progressive reduction of the anodic limits allows the synthesis of a non-overoxidised PANI film, as revealed by the absence of an anodic wave in the potential range $0.30-0.55 \mathrm{~V}$.
The so-obtained PANI films display the typical redox behaviour of this polymer, as illustrated in Fig. 2 for the films grown with 20 and 100 cycles. The reduction peak potential takes place at $0.06 \mathrm{~V}$ in both films while the thickest polymer exhibits a slightly higher oxidation peak potential ( 0.19 vs. $0.16 \mathrm{~V}$ for 20 cycles grown film) which is a characteristic feature of a thick film, also observed in other polymers [9]. The conversion reversibility $\left(Q_{\text {ox }} / Q_{\text {red }}\right)$ of both films is quite similar (1.05 and 1.02 for 20 and 100 growth cycles, respectively).

An interesting point relies on the fact that the charges involved in the redox processes are not proportional to the number of growth cycles. Indeed, oxidation charges, $Q_{\mathrm{ox}}$, of 0.41 and $4.40 \mathrm{mC} \mathrm{cm}^{-2}$ were measured for the thinnest and thickest film, respectively, which corresponds to a tenfold increase of the charge for a number of growth cycles five times higher. The high redox charges observed for the thicker film indicate the formation of a different structured layer. This polymer should display a structure allowing a more efficient charge transfer, likely due to a less compact morphology which facilitates the flux of ionic species taking place during the electrochemical processes.

The ellipsometric parameters depend on the electronic states of the film and the nature of the light interaction with these states. For the films studied in this work the ellipsometric data are displayed in Table
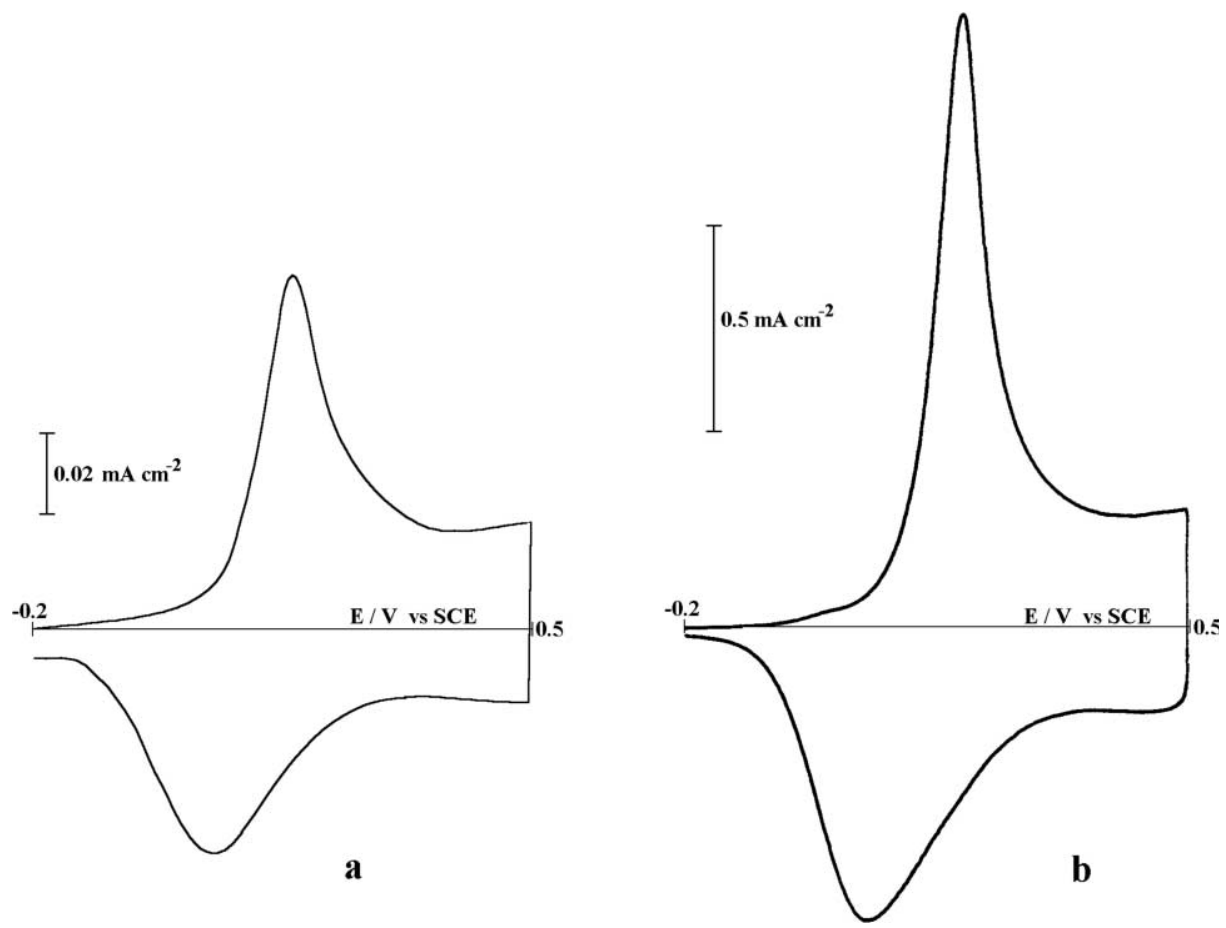

Fig. 2. Redox behaviour of PANI films potentiodynamically grown with (a) 20 and (b) 100 cycles. $0.5 \mathrm{M} \mathrm{H}_{2} \mathrm{SO}_{4} ; v=50 \mathrm{mV} \mathrm{s}^{-1}$. 
Table 1

Ellipsometric parameters of the PANI films deposited on gold coated silicon substrates

\begin{tabular}{lll}
\hline Number of growth cycles & $\psi$ & $\Delta$ \\
\hline 20 & 43.141 & 96.515 \\
50 & 42.603 & 89.081 \\
$20+30$ & 43.153 & 86.822 \\
100 & 46.690 & 64.525 \\
\hline
\end{tabular}

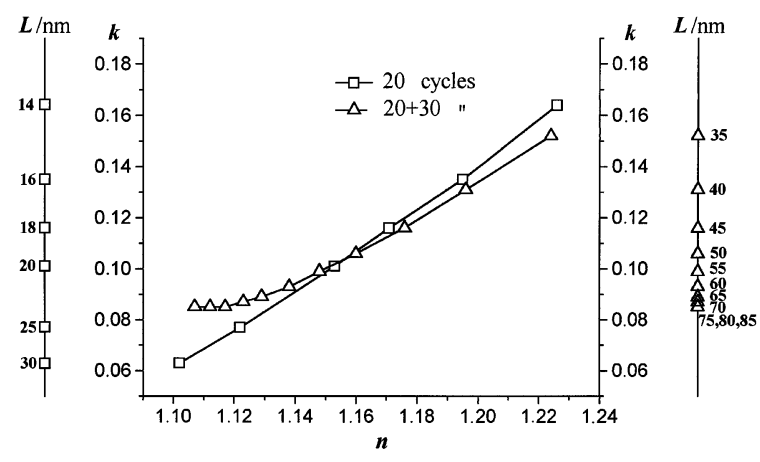

Fig. 3. Calculated values of $n$ and $k$ of two PANI films in the oxidised state.

1. The phase parameter is very sensitive to the thickness of the film, i.e. as the number of growth cycles increase, $\Delta$ decreases. It is interesting to note that films grown with 50 cycles using different procedures do not present the same value of $\Delta$. The film grown in two polymerisation sequences $(20+30$ cycles $)$ is therefore expected to be thicker (lower $\Delta$ ) than the polymer synthesised in a single procedure of 50 cycles (higher $\Delta$ ). As an amplitude parameter, $\psi$ responds mainly to the energy loss in the film and thus to the variations of the extinction coefficient of the polymer. The films grown with 20 and $20+30$ cycles present similar values of $\psi$, which suggests identical structures of these polymers. A slightly

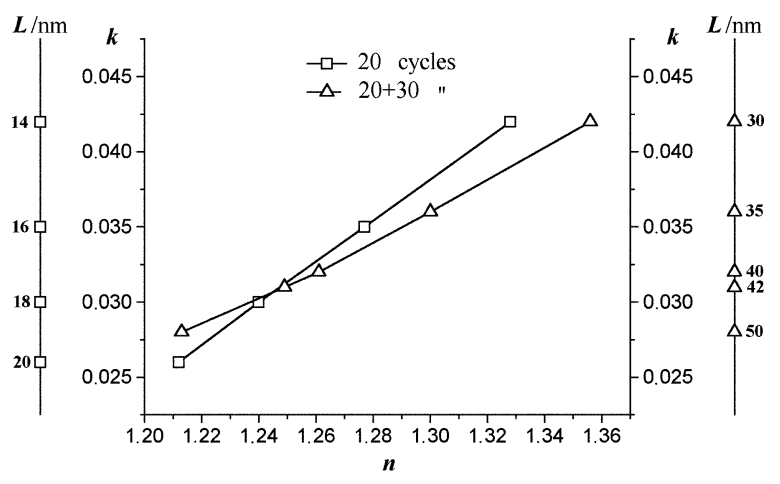

Fig. 4. Calculated values of $n$ and $k$ of two PANI films in the reduced state. different value of this parameter $\left(\sim 0.5^{\circ}\right)$ is observed for the layer grown with 50 consecutive cycles, indicating that the structure of this film should not be the same as the previous ones. A significant increase of $\psi$ was recorded for the thickest polymer, pointing out a different distribution of electronic states of the film, which should correspond to a different structured layer.

The ellipsometric parameters are function of the refractive index, $n$, extinction coefficient, $k$, and film thickness, $L$. As, by ellipsometry, only two experimental values are obtained, the determination of the three unknown requires a non-analytical approach. In this work, the evaluation of the optical properties of the films (which reflect the structure of the polymer) was performed by using a methodology which allows the simultaneous determination of $n, k$ and $L$ of two different thick films with the same structure. Assuming each film has a different thickness, the corresponding values of the refractive index and extinction coefficient were calculated; the plot of $n$ versus $k$ for the two films may exhibit a crossing point. In this case, they should present the same structure (same $n$ and $k$ ) and thickness correspondent to those employed for the calculation of these optical parameters.

The above described procedure was applied to the polymers grown with 20 and $20+30$ cycles, in the oxidised state (Fig. 3). The plot of $n$ versus $k$ of both films presents a crossing point at $n=1.156$ and $k=$ 0.104 . These optical parameters correspond to similarly structured layers with thicknesses estimated in $L=19.5$ and $51.3 \mathrm{~nm}$ for films grown with 20 and $20+30$ cycles, respectively. While the calculated value of the refractive index is comparable with those reported in literature, the extinction coefficient shows a lower value $(\tilde{n}=$ $1.125-0.354 \mathrm{i}$ [18] $\tilde{n}=1.17-0.20 \mathrm{i}$ [19]). This dissimilarity can be ascribed both to the use of different electrolyte solutions and to the fact that ellipsometric measurements, in this work, have been carried out ex situ, allowing the polymer to contact with air.

It has been reported by several authors that the polymer shrinks down upon reduction, and this phenomenon has been interpreted as due to the release of solvent and counterions [20]. Since the films of 20 and $20+30$ growth cycles present the same structure and the applied methodology permits to determine of the actual thickness of the polymer, it was also used with the same films, after polymer reduction (Fig. 4). In neutral form, the optical parameters of both polymers, determined at the point where the $n$ versus $k$ curves intercept, are $n=1.247$ and $k=0.031$, which corresponds to thicknesses of $L=17.5$ and $42.0 \mathrm{~nm}$ (20 and $20+30$ growth cycles, respectively). The film thickness difference in both redox states is due to a swelling, upon polymer doping, of $11-22 \%$, which is within the range of the reported values $(10-30 \%)$ in several studies of PANI [13,21,22]. Nevertheless, this result demon- 


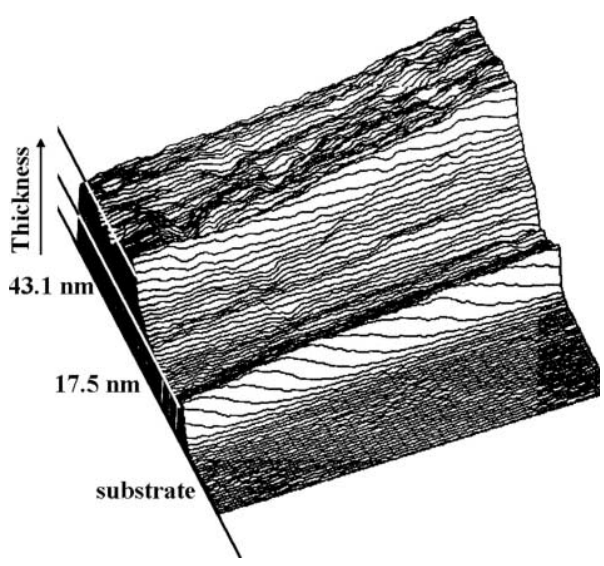

Fig. 5. Thickness distribution in 20 and $20+30$ growth cycles PANI sample in the reduced state. Lateral resolution $25 \times 30$ $\mu \mathrm{m}^{2}$.

strates that the extent of swelling depends on polymer thickness.

As expected, PANI in the reduced form is more compact (higher refractive index) and less absorbing (lower extinction coefficient) than in its oxidised state. Although $k$ displays a low value, it denotes that the film is not completely reduced. The same evidence is given by the value of $n$, which is $20 \%$ lower than expected for a fully reduced PANI film [18,19]. This observation indicates that the film oxidises to some extent when exposed to the air. It should be referred that, after the ellipsometric measurements, the polymer retained its optical properties without significant modification when stored in an inert atmosphere in the dry state for a period of, at least, $24 \mathrm{~h}$.
Additional support for the polymer thickness values calculated through the used methodology is provided by the imaging ellipsometry information. Fig. 5 displays the thickness distribution of the polymer in the 'stair' sample with 20 and $20+30$ growth cycles in the reduced state. Since the $y$-axis is directly proportional to the thickness, assuming $L=17.5 \mathrm{~nm}$ for the 20 growth cycles film, a thickness of $43.1 \mathrm{~nm}$ results for the polymer with $20+30$ growth cycles. The small difference of the thickness values determined by different approaches (less than 3\%), gives credit to the calculated characteristic parameters of the films.

Since the polymer grown with $20+30$ cycles was subjected to a voltammetric characterisation in a monomer-free solution after 20 growth cycles, its properties should be compared with the film grown with the same number of cycles in a single potentiodynamic sequence. Fig. 6 contrasts the redox behaviour of films synthesised with 50 cycles using the different procedures. It is noticed that the film grown by two potentiodynamic sequences up to 50 cycles displays a higher electroactivity $\left(E_{\mathrm{p}} \mathrm{ox}=0.16 \mathrm{~V} ; Q_{\mathrm{ox}}=1.14 ; Q_{\mathrm{red}}=1.10\right)$ than the polymer synthesised by a single operation $\left(E_{\mathrm{p}} \mathrm{ox}=0.18 \mathrm{~V} ; Q_{\mathrm{ox}}=0.99 ; Q_{\mathrm{red}}=0.93\right)$. These results point to a higher compactness (more difficult redox conversion) of the film prepared with 50 consecutive cycles, as already suggested by the relatively high value of $\Delta$ (film thinner than the one grown with $20+30$ cycles). It is so expected that the electrochemical characterisation (five cycles) of the film prepared with 20 growth cycles produces structural modifications, which influences the subsequent growth, due to the memory effect. Indeed, the plot of $n$ versus $k$ calculated with reasonable values of thickness for these films does not present a cross in a range of optical values with physi-

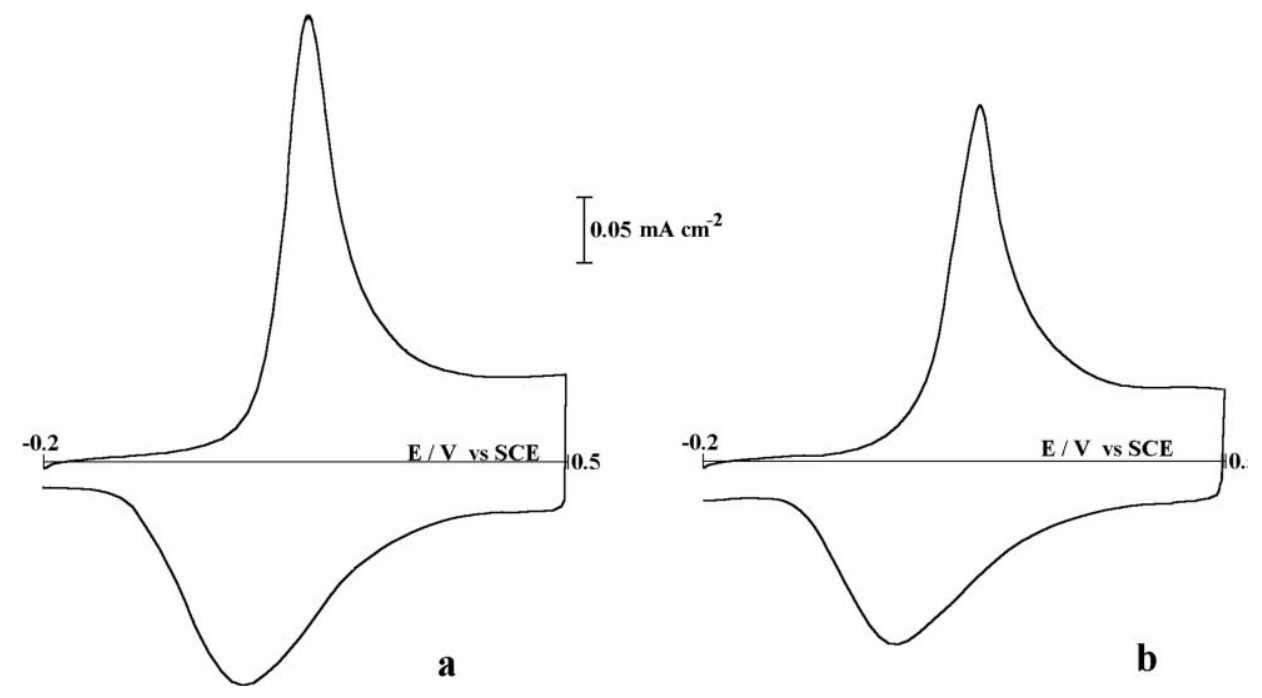

Fig. 6. Redox behaviour of PANI films potentiodynamically grown with (a) $20+30$ and (b) 50 cycles. $0.5 \mathrm{M} \mathrm{H}_{2} \mathrm{SO}_{4} ; v=50 \mathrm{mV} \mathrm{s}-1$. 


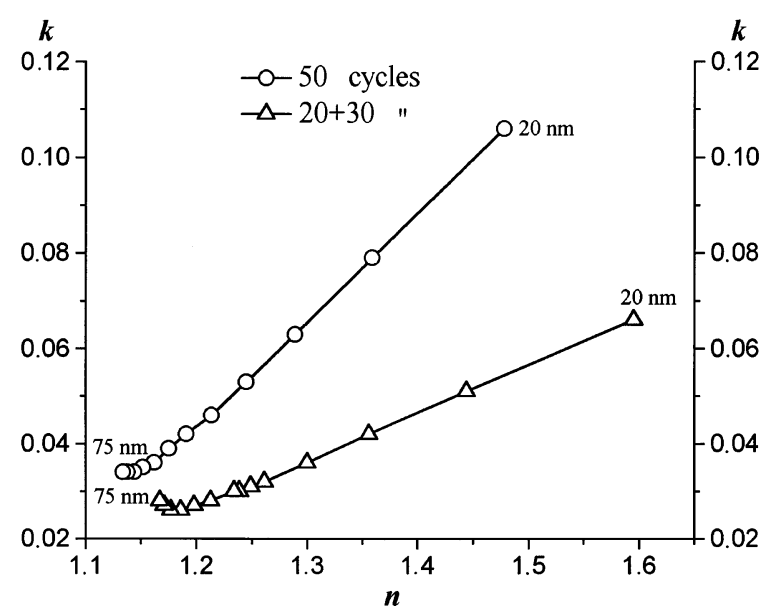

Fig. 7. Calculated values of $n$ and $k$ of two PANI films grown with 50 and $20+30$ cycles (reduced state).

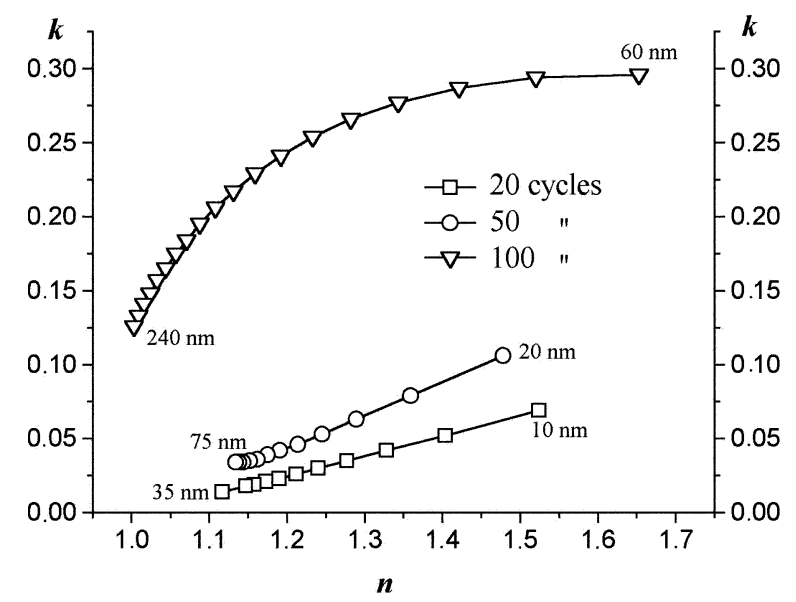

Fig. 8. Calculated values of $n$ and $k$ of two PANI films (reduced state) grown with 20, 50 and 100 cycles. The assumed thickness used to compute the values of $n$ and $k$ is indicated. cal meaning (Fig. 7). This observation clearly reveals that different polymerisation procedures give rise to distinctly structured films.

Fig. 8 is represents the $n$ versus $k$ plots (calculated assuming different thickness values) of different thick films grown in a single potentiodynamic sequence. As expected, considering the ellipsometric parameters and the electrochemical data retrieved from the redox behaviour analysis of these polymers (Figs. 2 and 6a), they present different structures (absence of interception points). Nevertheless, it is noticed that the two thinnest films display a linear dependence of $n$ versus $k$ whilst, for the layer grown with 100 cycles, $k$ tends to a constant value (as $L$ decreases) being related with $n$ by a different function. Considering the optical parameters' distinct response to the thickness change (reported to the experimental ellipsometric data), a two-layered formation can be envisaged for thick polymers.

Tridimensional representations, obtained by imaging ellipsometry, of the thickness distribution of PANI films grown with 20 and 50 cycles are shown in Fig. 9. These experiments revealed that both films have a homogeneous distribution of the thickness in all the area. Taking into account that the $y$-axis resolution of Fig. 9a and $\mathrm{b}$ are not equal due to the differences of film thickness, the surface morphology of the thick polymer appears to have a higher degree of roughness, as it was expected for a polymer with a more open structure.

\section{Conclusions}

The use of ellipsometry to characterise PANI films potentiodynamically prepared on gold electrodes allows us to observe structural differences among films with distinct thicknesses. To obtain equivalent and reproducible PANI films, a careful electrochemical approach is required. Evidence is given that the doping/undoping process of a given layer produces irreversible structural modifications. Films grown with 50 cycles, which were electrochemically characterised after the first 20 cycles
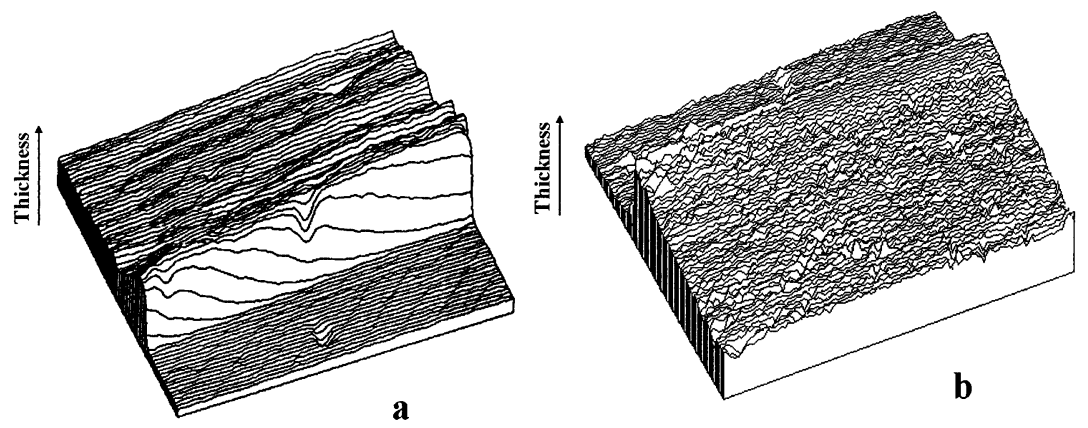

Fig. 9. Thickness distribution of PANI films grown with (a) 20 and (b) 100 cycles. Lateral resolution $25 \times 30 \mu \mathrm{m}^{2}$. 
of growth, have the same structure than a 20 growth cycles polymer. These films, in the dry state, are optically characterised by a complex refractive index at $\lambda=632.8 \mathrm{~nm}$ of $\tilde{n}=1.156-0.104 \mathrm{i}$ and $\tilde{n}=1.247-$ $0.031 \mathrm{i}$ in the oxidised and reduced forms, respectively.

Combining experimental ellipsometric data $(\psi, \Delta)$ of several PANI films a procedure was applied to evaluate the refractive index, extinction coefficient and thickness of each film with the same structure. Imaging ellipsometry, which informs on thickness distribution and surface morphology, provides good support to the applied methodology. This approach permitted the evaluation of $11-22 \%$ swelling of thin PANI films upon doping.

Results point to the formation of PANI films with a uniform thickness distribution of the polymer (regardless of the number of growth cycles), thick films being (100 cycles of growth) more porous than thin ones.

\section{References}

[1] L. Groenendaal, F. Jonas, D. Freitag, H. Pielartzik, J.R. Reynolds, Adv. Mater. 12 (2000) 481.

[2] A. Galal, J. Solid State Electrochem. 2 (1998) 7.

[3] G. Inzelt, M. Pineri, J.W. Schultze, M.A. Vorotyntsev, Electrochim. Acta 45 (2000) 2403.

[4] Y.M. Volkovich, A.G. Sergeev, T.K. Zolotova, S.D. Atanasiev, O.N. Efimov, E.P. Krinichnaya, Electrochim. Acta 44 (1999) 1543.

[5] H. Tang, A. Kitani, M. Shiotani, Electrochim. Acta 41 (1996) 1561.
[6] G. Inzelt, Electrochim. Acta 45 (2000) 3865.

[7] P.A. Kilmartin, G.A. Wright, Electrochim. Acta 41 (1996) 1677.

[8] B.R. Scharifker, D.J. Fermin, J. Electroanal. Chem. 365 (1994) 35.

[9] L.M. Abrantes, J.P. Correia, Electrochim. Acta 44 (1999) 1901.

[10] F. Chao, M. Costa, E. Museux, E. Levart, L.M. Abrantes, J. Chim. Phys. 89 (1992) 1009.

[11] L. Duic, Z. Mandic, S. Kovac, Electrochim. Acta 40 (1995) 1681.

[12] C. Zhang, B. Yao, J. Huang, X. Zhou, J. Electroanal. Chem. 440 (1997) 35.

[13] C.M.G.S. Cruz, E.A. Ticianelli, J. Electroanal. Chem. 428 (1997) 185.

[14] R. Yang, D.F. Evans, L. Christensen, W.A. Hendrickson, J. Phys. Chem. 94 (1990) 6117.

[15] C. Tian, G. Jin, F. Chao, M. Costa, J.P. Roger, Thin Solid Films 233 (1993) 91.

[16] G. Jin, R. Jansson, I. Lundström, H. Arwin, in: Imaging Ellipsometry for Biosensor Applications, Transducters'95. Eurosensors IX, Stockolm, 1995.

[17] G. Jin, R. Jansson, H. Arwin, Rev. Sci. Instrum. 67 (1996) 2930.

[18] R. Greef, M. Kalaji, L.M. Peter, Faraday Discuss. Chem. Soc. 88 (1989) 277.

[19] S. Gottesfeld, A. Redondo, S.W. Feldberg, J. Electrochem. Soc. 134 (1987) 271.

[20] C.M. Carlin, L.J. Kepley, A.J. Bard, J. Electrochem. Soc. 132 (1985) 353.

[21] H. Arwin, R. Jansson, Electrochim. Acta 39 (1994) 211.

[22] C. Barbero, R. Kotz, J. Electrochem. Soc. 141 (1994) 859. 